\title{
Complete response in hepatocellular carcinoma with lymph node metas- tasis by combination therapy of atezolizumab and bevacizumab: a case report
}

\author{
Sang Youn Hwang, Sun Mi Lee, Jeong Woo Lim, Gi Jung Jeon, Hye Won Lee \\ Department of Internal Medicine, Dongnam Institute of Radiological \& Medical Sciences, Busan, Korea
}

Received Aug. 6, 2021

Revised Aug. 25, 2021

Accepted Sep. 10, 2021
Sorafenib is the oldest first line systemic treatment in patients with advanced hepatocellular carcinoma (HCC) and has been used exclusively for nearly 10 years. The superiority of administering a combination of atezolizumab plus bevacizumab (AteBeva) compared to sorafenib as first line systemic treatment for unresectable HCC was recently proven during the IMbrave150 Phase III randomized trial. While clinicians can expect improved responses and treatment outcomes due to the good results of the IMbrave 150 trial, they must also consider that atezolizumab can cause various immune-related adverse events (IrAEs). Based on the above suggestions, we herein present a case of HCC with lymph node metastasis who achieved complete remission following treatment with AteBeva and developed an IrAE (adrenal insufficiency). Further study of real-life data regarding combination therapy with AteBeva is needed to manage patients with advanced HCC. (J Liver Cancer 2021;21:177-180)

Keywords: Case report; Hepatocellular carcinoma; Atezolizumab; Bevacizumab; Adrenal insufficiency

\section{INTRODUCTION}

Sorafenib is the oldest first line therapy for advanced hepatocellular carcinoma (HCC) and has been used exclusively for nearly 10 years since several trials trying to prove the superiority of candidate drugs over sorafenib have failed. ${ }^{1,2}$ Only lenvatinib was approved as an additional first line therapy for advanced HCC following the success of the REFLECT trial; however, the overall survival (OS) was non-in-

\section{Corresponding author: Sang Youn Hwang}

Department of Internal Medicine, Gastrointestinal Cancer Center, Dongnam Institute of Radiological \& Medical Sciences, 40 Jwadong-gil, Jangan-eup, Busan 46033, Korea

Tel. +82-51-720-5114, Fax. +82-51-720-5992

E-mail: mongmani@hanmail.net ferior to that of sorafenib. ${ }^{3}$ Recent research proved that the OS and tumor response of combination therapy of atezolizumab plus bevacizumab (AteBeva) was superior to that of sorafenib; therefore, clinicians can expect a better response and treatment outcome. However, clinicians must also consider that atezolizumab may cause several immune-related adverse events (IrAEs). According to the above suggestions, we report a case of HCC with lymph node (LN) metastasis that achieved complete remission (CR) following AteBeva combination therapy who developed an IrAE (adrenal insufficiency). This case report has been described according to the CARE guidelines available from https://www.care-statement.org/. 


\section{CASE REPORT}

A 74-year-old male patient was transferred to our hospital for additional treatment of HCC, and portocaval and precaval LN metastasis were observed during computed tomography (CT) after one session of transarterial chemoembolization (TACE) for marginal recurrence in another cancer center (Fig. 1A, 2A). He underwent subtotal gastrectomy for gastric cancer in 2017 and was subsequently diagnosed with single HCC (Barcelona Cancer Liver Clinic [BCLC] stage A) and alcoholic liver cirrhosis with Child-Pugh class A liver function. He underwent laparoscopic wedge resection in
2019. There is no family history of note. The initial laboratory test revealed a hemoglobin level of $13.0 \mathrm{~g} / \mathrm{dL}$ and platelet and white blood cell counts of $152 \times 10^{6} / \mathrm{L}$ and $9.44 \times 10^{9} / \mathrm{L}$, respectively. The liver function test results were within normal limits. The level of alpha-fetoprotein (AFP) was 1,584.6 $\mathrm{ng} / \mathrm{mL}$, while that of protein induced by vitamin $\mathrm{K}$ absence or antagonist-II (PIVKA-II) was 2,657 mAU/mL.

Esophageal varices with $\mathrm{F} 1 \mathrm{Cw} \mathrm{RC}(-)$ according to the Japanese Research Society for Portal Hypertension guidelines were observed during endoscopic evaluation. We diagnosed the patient with HCC (BCLC C) and defined him as a TACE-refractory case due to stage progression from BCLC B
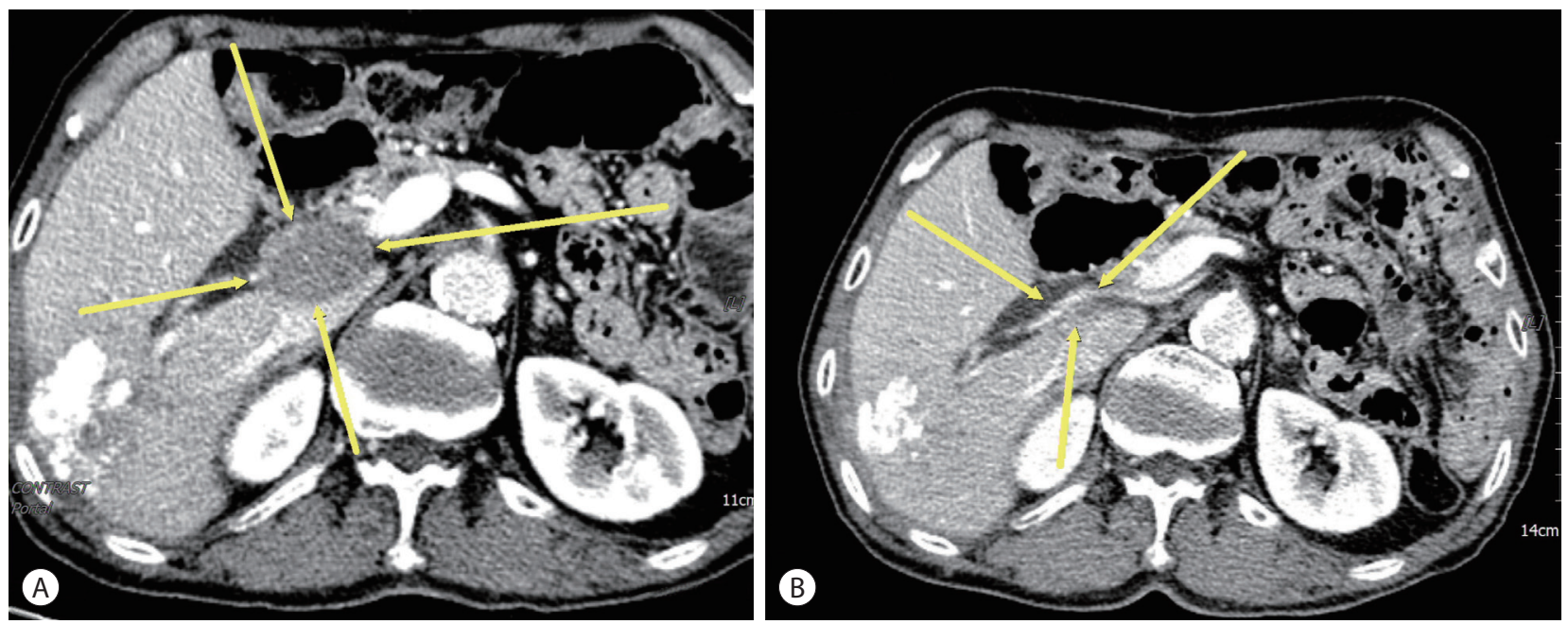

Figure 1. Liver computed tomography findings. (A) $3.7 \times 2.6 \mathrm{~cm}$ portocaval lymph node (LN) enlargement was noted (arrows). (B) Previous portocaval LN enlargement had nearly disappeared after six cycles of combination therapy of atezolizumab plus bevacizumab (AteBeva) was administered (arrows).
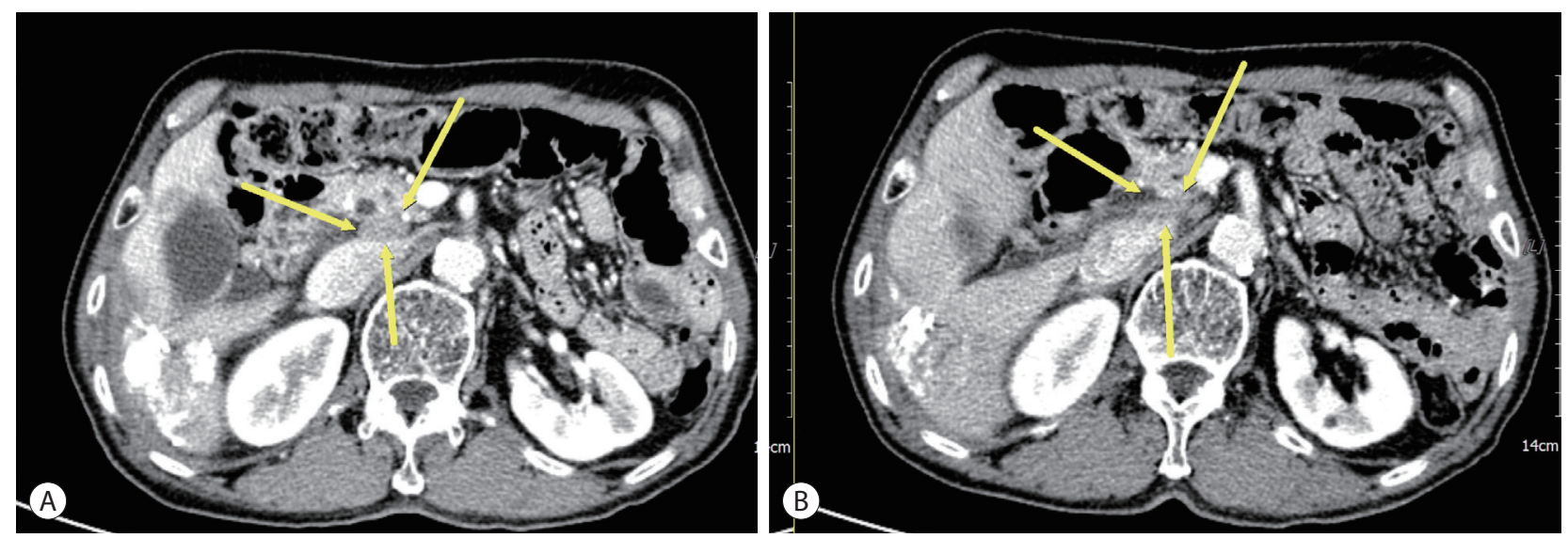

Figure 2. Liver computed tomography findings. (A) $2.1 \times 1.0 \mathrm{~cm}$ precaval lymph node (LN) enlargement was noted (arrows). (B) Previous precaval LN enlargement had nearly disappeared after six cycles of combination therapy of atezolizumab plus bevacizumab (AteBeva) (arrows). 
to $\mathrm{C}$ caused by new-onset LN metastasis. We decided to administer systemic therapy (atezolizumab, 1,200 mg, Roche, Basel, Swiss; bevacizumab, $15 \mathrm{mg} / \mathrm{kg}$, Roche, Basel, Swiss) to the patient at 3-week intervals. After 6 cycles of systemic therapy, LN metastases had nearly disappeared in follow up CT (Fig. 1B, 2B) and the AFP level was normalized (1,584.6 $\mathrm{ng} / \mathrm{mL} \rightarrow 3.0 \mathrm{ng} / \mathrm{mL})$, as was that of PIVKA-II $(2,657 \mathrm{mAU} /$ $\mathrm{mL} \rightarrow 20 \mathrm{mAU} / \mathrm{mL})$. However, after 9 cycles of systemic therapy, the patient presented to our clinic complaining of general weakness and a confused mental state. A serum sodium level of $109 \mathrm{mmol} / \mathrm{L}$ was noted. Because the patient did not use steroids previously, we suspected adrenal insufficiency associated with autoimmune adrenalitis due to atezolizumab administration and performed an adrenocorticotropic hormone (ACTH) stimulation test. The results were as follows: cortisol levels at baseline, 30, and 60 minutes after ACTH injection were $3.6 \mu \mathrm{g}, 7.5 \mu \mathrm{g}, 9.6 \mu \mathrm{g}$, respectively. We then confirmed adrenal insufficiency and administered oral hydrocortisone. The patient's serum sodium level normalized after 10 days. The patient receives combination treatment continuously and we follow up the patient carefully.

The present case report was approved by the Institutional Review Board (IRB) of the Dongnam Institute of Radiological \& Medical Sciences, Busan, Korea (IRB number: D-2108019-002) and the patient informed consent was obtained. The study protocol conformed to the ethical guidelines of the Declaration of Helsinki of the World Medical Association.

\section{DISCUSSION}

The superiority of combination therapy with AteBeva compared with sorafenib as a first line systemic treatment for unresectable HCC has been proven in the IMbrave150 Phase III randomized trial. The one-year OS rates of the AteBeva $\operatorname{arm}(67.2 \%$; 95\% confidence interval [CI], 61.3-73.1) were significantly greater than those in the sorafenib arm $(54.6 \%$; 95\% CI, 45.2-64.0), and the median progression-free survival was 6.8 (95\% CI, 5.7-8.3) and 4.3 months (95\% CI, 4.0-5.6) in the respective groups (hazard ratio [HR] for disease progression or death $=0.59 ; 95 \% \mathrm{CI}, 0.47-0.76 ; P<0.001)$. The overall objective response rates of AteBeva and sorafenib were $27.3 \%$ versus $11.9 \%$ (by RECIST v1.1) and 33.2\% versus $13.3 \%$ (by modified RECIST), respectively. Moreover, the median time to deterioration of patient-reported quality of life was 11.2 months with AteBeva versus 3.6 months with sorafenib (HR, 0.63; 95\% CI, 0.46-0.85). ${ }^{4}$ A recently updated OS analysis of IMbrave150 data reported that the median OS of AteBeva and sorafenib were 19.2 and 13.4 months at 12 months of additional follow-up after the primary analysis, respectively. ${ }^{5}$ The theoretical background of the synergistic effect of AteBeva is the reduction of vascular endothelial growth factor-mediated immunosuppression and promotion of T-cell infiltration within the tumor and its microenvironment by bevacizumab, along with the enhancement of antiprogrammed death (PD)-1 and anti-PD-L1 antitumor effects. ${ }^{4}$

Immune checkpoint inhibitors (ICPIs) can induce several immune-related irAEs that are usually manageable, but occasionally life-threatening. IrAEs are unrelated to the dose, and have an incidence of $27 \%$ for all grades and $6 \%$ for grade 3 or higher. The rates of adrenal insufficiency due to ICPIs are $1-3 \%$ and clinicians must consider adrenal dysfunction if symptoms such as fatigue, hypotension, nausea, vomiting, and laboratory findings such as hyponatremia are present in patients treated with ICPIs. ${ }^{6}$

The limitation of this case report is that we could not definitively prove that autoimmune adrenalitis was caused by atezolizumab, because we did not check cortisol levels before AteBeva therapy was administered, and could not observe the typical radiologic traits ${ }^{7}$ of autoimmune adrenalitis, such as bilateral enlargement of the adrenal glands, following AteBeva therapy. However, we had enough evidence to suspect adrenal insufficiency due to atezolizumab administration because of the sudden onset of symptoms such as hyponatremia, confused mental state etc. after several cycles of AteBeva therapy in our case report.

In conclusion, we administered combination therapy of AteBeva as a first-line systemic treatment for a patient with HCC and LN metastases who attained CR. Moreover, we diagnosed and treated adrenal insufficiency during treatment. To the best of our knowledge, cases that attain CR after AteBeva administration have not been reported in Korea to 
date and we have offered our experience herein. We need to observe further real-life data in Korea regarding combination therapy of AteBeva.

\section{Conflicts of Interest}

The authors have no conflicts of interest to disclose.

\section{Ethics Statement}

The present case report was approved by the Institutional Review Board (IRB) of the Dongnam Institute of Radiological \& Medical Sciences, Busan, Korea (IRB number: D-2108019-002). Informed consent was obtained from the patient. The study protocol conformed to the ethical guidelines of the Declaration of Helsinki of the World Medical Association.

\section{Funding Statement}

No funding to declare.

\section{Data Availability}

All data generated or analyzed during the study are included in this published article.

\section{ORCID}

Sang Youn Hwang https://orcid.org/0000-0003-2750-9379

Sun Mi Lee https://orcid.org/0000-0002-5770-4477

Jeong Woo Lim https://orcid.org/0000-0002-7182-9962

Gi Jung Jeon https://orcid.org/0000-0001-6777-6055

Hye Won Lee https://orcid.org/0000-0002-8362-442X

\section{Author Contribution}

Conceptualization: HSY

Data curation: JKJ

Methodology: IJW

Project administration: LSM

Writing original draft: LHW

Writing review \& editing: HSY, LHW, LSM, IJW, JKJ

Approval of final manuscript: all authors.

\section{References}

1. Cheng AL, Guan Z, Chen Z, Tsao CJ, Qin S, Kim JS, et al. Efficacy and safety of sorafenib in patients with advanced hepatocellular carcinoma according to baseline status: subset analyses of the phase III Sorafenib Asia-Pacific trial. Eur J Cancer 2012;48:14521465.

2. Bruix J, Raoul JL, Sherman M, Mazzaferro V, Bolondi L, Craxi A, et al. Efficacy and safety of sorafenib in patients with advanced hepatocellular carcinoma: subanalyses of a phase III trial. J Hepatol 2012:57:821-829.

3. Kudo M, Finn RS, Qin S, Han KH, Ikeda K, Piscaglia F, et al. Lenvatinib versus sorafenib in first-line treatment of patients with unresectable hepatocellular carcinoma: a randomised phase 3 noninferiority trial. Lancet 2018;391:1163-1173.

4. Finn RS, Qin S, Ikeda M, Galle PR, Ducreux M, Kim TY, et al. Atezolizumab plus bevacizumab in unresectable hepatocellular carcinoma. N Engl J Med 2020;382:1894-1905.

5. Finn RS, Qin S, Ikeda M, Galle PR, Ducreux M, Kim T, et al. IMbrave150: Updated overall survival (OS) data from a global, randomized, open-label phase III study of atezolizumab (atezo) + bevacizumab (bev) versus sorafenib (sor) in patients (pts) with unresectable hepatocellular carcinoma (HCC). J Clin Oncol 2021;39 suppl:267.

6. Sangro B, Chan SL, Meyer T, Reig M, El-Khoueiry A, Galle PR. Diagnosis and management of toxicities of immune checkpoint inhibitors in hepatocellular carcinoma. J Hepatol 2020;72:320-341.

7. Chang LS, Barroso-Sousa R, Tolaney SM, Hodi FS, Kaiser UB, Min $L$. Endocrine toxicity of cancer immunotherapy targeting immune checkpoints. Endocr Rev 2019;40:17-65. 